\title{
Heat (Mass) Transfer Distributions in a Rotating Two-Pass Channel with Angled Ribs
}

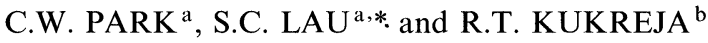 \\ ${ }^{a}$ Department of Mechanical Engineering, Texas A\&M University, College Station, TX 77843-3123, USA; \\ ${ }^{\mathrm{b}}$ Lynntech Inc., 7610 Eastmark Drive, College Station, TX 77840, USA
}

(Received 26 February 1998; In final form 12 March 1998)

\begin{abstract}
Experiments have been conducted to study the local heat (mass) transfer distributions in a two-pass test model of internal turbine blade cooling passages, with $60^{\circ}$ ribs on the leading and trailing walls. For radial outward flow in the first pass, rotation did not significantly increase the local nor the overall heat (mass) transfer between consecutive ribs on the trailing wall. Rotation-induced Coriolis force lowered the relative overall heat (mass) transfer on the leading wall less in the rib-roughened channel than in a smooth channel. When the rotation number was high, there were twin peaks in the local heat (mass) transfer distribution between ribs on the leading wall. For radial inward flow in the second pass, the sharp turn reduced the difference between the heat (mass) transfer on the leading wall and that on the trailing wall.
\end{abstract}

Keywords: Rotating two-pass channel, Angled ribs, Naphthalene sublimation

\section{INTRODUCTION}

Airfoils in modern gas turbine engines are cooled with air that flows through rib-roughened serpentine channels, and bleeds through film cooling holes. The flow patterns in these internal channels and the heat transfer distributions on the channel walls are very different from those in stationary straight channels with smooth walls. In a cooling channel in a gas turbine airfoil, Coriolis force pushes the airflow toward various walls, depending on the flow direction and the orientations of the walls with respect to the rotating axis. Crossstreamwise variation of the buoyancy force causes radial secondary flow. Centrifugal force in a sharp turn induces flow impingement on the various walls at the turn. Ribs force spatially periodic flow separation and reattachment. With angled ribs, near-wall secondary flows along the directions of the rib axes enhance the asymmetry of the boundary layers near the ribbed walls. The interactions among the forces in the flow due to rotation, sharp turns, and ribs result in large variations of the local heat transfer coefficient on the walls of an internal cooling channel in a turbine airfoil.

The dimensionless parameters governing the flow field in a rotating frame are the Reynolds number, the rotation number, and a buoyancy

*Corresponding author. Tel.: (409) 845-0171. Fax: (409) 862-2418. E-mail: slau@)mengr.tamu.edu. 
parameter. These parameters may be considered as ratios of the inertial, viscous, Coriolis, and buoyancy forces. Factors that affect the heat transfer distribution, for air flow through a rotating multipass channel, also include the flow entrance conditions, the thermal boundary conditions, the main flow direction (radial outward or inward), the channel shape and orientation, the geometries of the turns, and the rib configuration.

Extensive heat transfer results are available in the literature for straight and multipass, rib-roughened channels, rotating about perpendicular axes. Published results were reviewed in recent publications such as Tse and Steuber (1996) and Park et al. (1998). Wagner et al. (1992) and Johnson et al. $(1993,1994)$ investigated the heat transfer in multipass square channels with ribs normal and skewed to the flow. Johnson et al. (1993) also studied the effect of model orientation on the heat transfer in rotating multipass square channels with ribbed walls. Parsons et al. (1994) and Zhang et al. (1995) reported results on the effect of varying the wall temperatures on the heat transfer in a two-pass square channel with normal and angled ribs.

It should be apparent that almost all of the published experimental results on heat transfer in rotating channels are regional average results. In this investigation, the detailed local heat transfer distributions on the walls of a rotating, two-pass, square channel, with $60^{\circ}$ ribs on the leading and trailing walls, are determined using the naphthalene sublimation technique and the heat/mass transfer analogy (Goldstein and Cho, 1995). The test channel models the cooling channels in turbine airfoils. Attention is focused on the heat (mass) transfer distributions on the leading and trailing walls of the test channel. Since the maximum variation of the density of the naphthalene vapor-air mixture that passed through the test channel during any experiment was only about $0.05 \%$, the density variation effect was negligible. Therefore, this investigation examines only the effects of the Coriolis force, the turn, and the ribs, but not the buoyancy force, on the local heat (mass) transfer distributions. The detailed local results of this investigation will enable better understanding of the effects of rotation, a sharp turn, and angled ribs on the local heat (mass) transfer distribution in a square channel, and will help improve the design of serpentine cooling channels in turbine airfoils.

\section{TEST APPARATUS AND PROCEDURE}

The test section was a two-pass channel with a sharp $180^{\circ}$ turn, constructed entirely of alumin' $\mathrm{m}$. It had a $1.59 \mathrm{~cm}$ by $1.59 \mathrm{~cm}$ square flow cross section. The two straight segments are $0.11 \mathrm{~m}$ long, or seven-hydraulic-diameter long. The test section had seven separate walls: two principal walls, two inner walls (each of which is one-half of the wall that separates the two straight segments), two outer side walls, and an end wall (see Fig. 1). Naphthalene in shallow cavities covered the inner surfaces of the walls. Once these walls were assembled for an experiment, the interior surfaces of the test section were all mass transfer active, and the total thickness of the two inner walls was one-half of the channel hydraulic diameter or the channel width.

Ribs were cut from $1.59 \mathrm{~mm}$ by $1.59 \mathrm{~mm}$, square, balsa wood strips. Therefore, the height of the ribs was one-tenth the channel height or the hydraulic diameter. The ribs were attached with epoxy on the two straight segments of the two principal walls. The angle between the axis of each rib and the main flow direction was $60^{\circ}$. The rib arrays on the two opposite principal walls were aligned and parallel. The rib pitch was equal to the hydraulic diameter of the test channel. The ribs were coated with naphthalene with a brush.

Figure 2 shows how the seven walls of the test section were assembled inside a rectangular aluminum housing that had two parallel square channels separated by a $0.79 \mathrm{~cm}$ thick wall. Once the seven individual walls were secured inside this housing, the assembly consisted of an entrance channel, the two-pass test channel, and an exit channel. The entrance and exit channels had the same square cross section as the test section, and had lengths of 10 and 20 hydraulic diameters, 


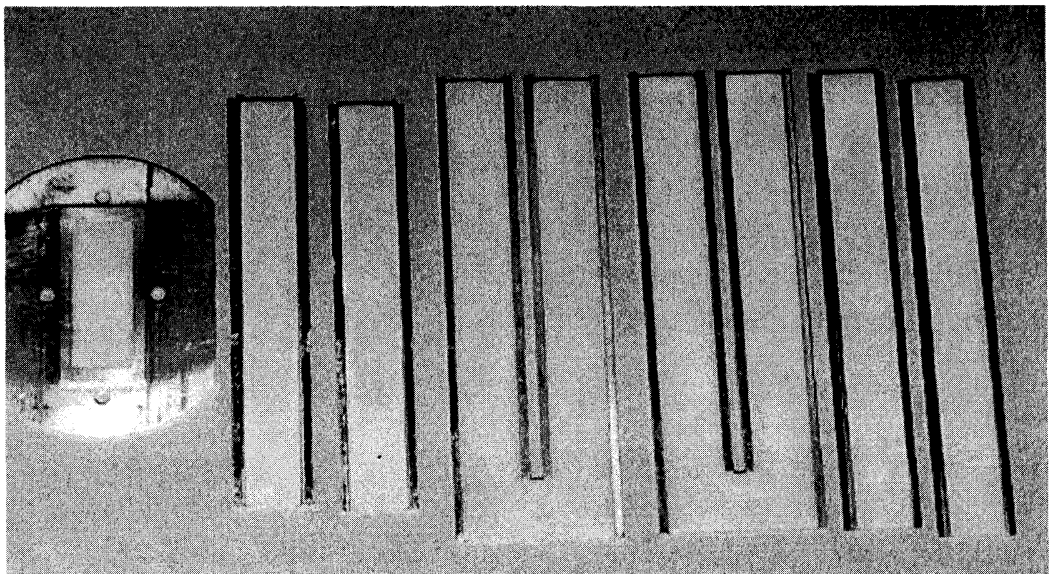

FIGURE 1 Separate naphthalene-coated walls of the test section.

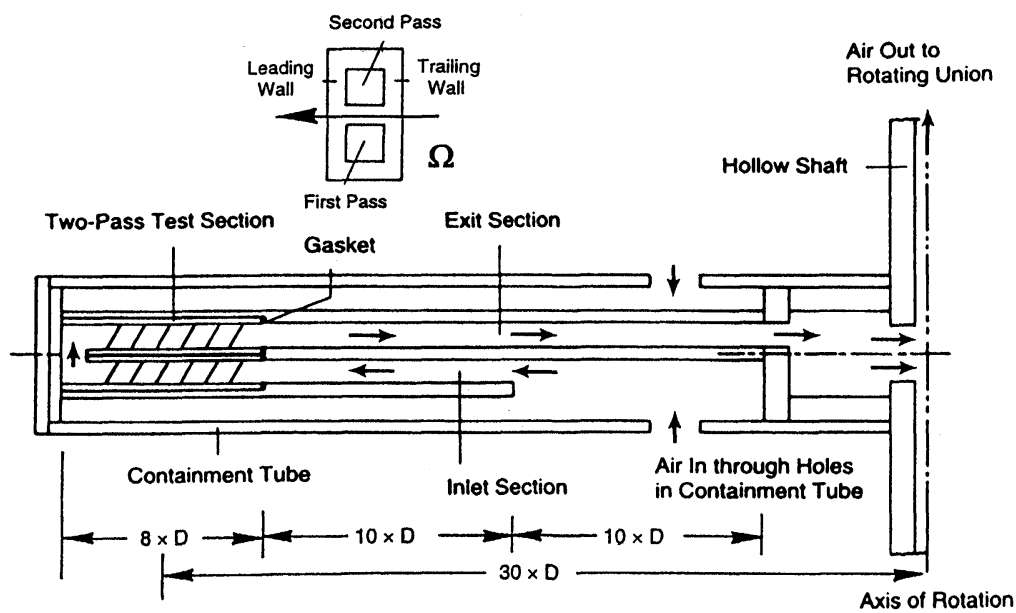

FIGURE 2 Schematic of the two-pass test channel in the entrance/test/exit channel assembly.

respectively. The end of this assembly with the outlet of the exit channel was welded to an aluminum disk. The disk was attached with screws to one end of a short horizontal aluminum tube that was affixed to the vertical steel shaft of a rotation test rig, such that the mean rotating radius of the test section was 30 times the channel hydraulic diameter.

The vertical rotating shaft was supported by two bearings in a cage that was constructed of two large $0.64 \mathrm{~cm}$ thick steel plates and heavy steel square tubes (see Fig. 3). Steel mesh, reinforced with steel angles and thick plywood boards, fenced the vertical faces of the steel cage. A five-horsepower electric motor drove the shaft with a belt and two pulleys. As shown in Fig. 2, an aluminum containment tube enclosed the entire entrance/test/exit channel assembly. The entrance/test/exit channel assembly and the containment tube were balanced by a second aluminum tube of the same diameter as the containment tube. The latter aluminum tube was affixed to the other end of the short horizontal aluminum tube in the middle and was equipped with an adjustable sliding counterweight. 


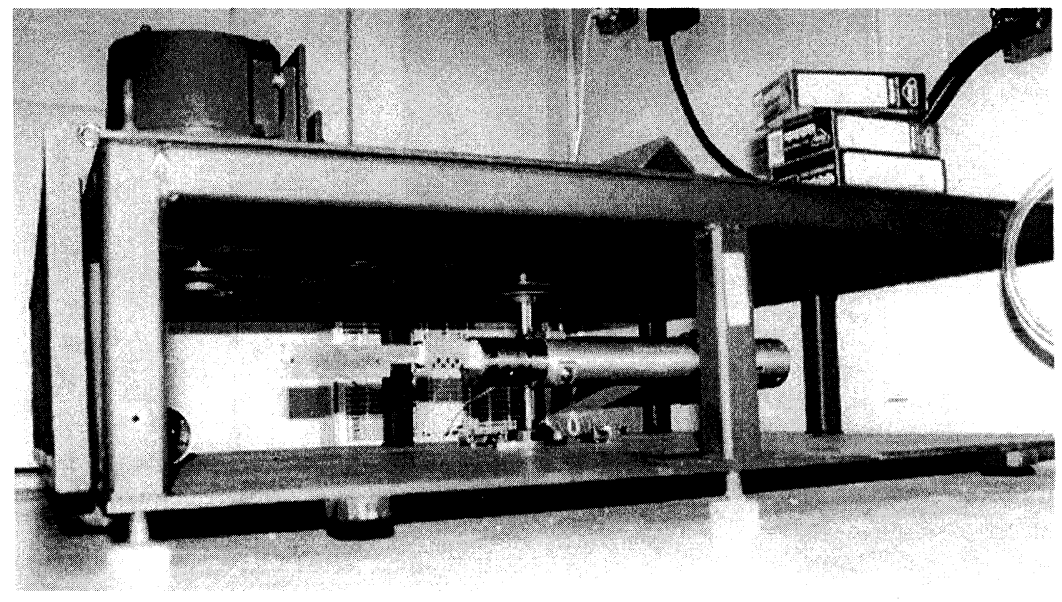

FIGURE 3 The rotation test rig

Air at room temperature was drawn through bleed holes in the containment tube and then the entrance/test/exit section assembly with a centrifugal blower. The air then flowed through the hollow rotating shaft, a rotating union, a calibrated orifice flow meter, and a gate valve, before it was ducted to an exhaust hood.

To obtain the distributions of the local mass transfer coefficient on the two principal walls of the test section, the elevations at a grid of up to about 750 points on the surface of each wall were measured and recorded at the beginning and the end of each test run. To measure the surface contour on a test wall, the wall was affixed firmly on an $X-Y$ coordinate table that was equipped with stepper motors to traverse the test wall in a horizontal plane. An electronic depth gage with a resolution of $2.5 \times 10^{-4} \mathrm{~mm}$ was used to measure the surface elevations at the grid points. The depth gage consisted of a lever-type sensor connected to an electronic amplifier. The stepwise movement of the coordinate table and the recording of output data were controlled with a microcomputer that was equipped with an A/D board.

An electronic balance with a resolution of either 0.01 or $0.1 \mathrm{mg}$ was used to weigh the seven walls. Two manometers measured the pressures at the orifice flow meter, and thermocouples measured the inlet air temperature and the air temperature at the orifice flow meter. An electronic tachometer was used to measure the rotational speed.

At the beginning of a test run, all seven walls of the test channel were weighed and the initial elevations at the grid of points on the naphthalene-coated surface of each of the two principal walls were obtained. The individual walls of the test section were then quickly secured in the entrance/ test/exit channel assembly in the containment tube. It should be pointed out that the test section was designed so that the seven walls could be assembled (or disassembled) very quickly to minimize extraneous mass losses from the naphthalene-coated surfaces of the walls.

After the motor was switched on to rotate the test section at the desired angular velocity, air was drawn through the test section with the blower. A test run lasted one to two hours. During the run, the air flow rate and the air temperatures were monitored and recorded periodically. At the end of the run, the walls of the test section were removed from inside the containment tube. Each wall was weighed and the elevations at the same grid of points were measured again.

Separate experiments were carried out to determine the correction to the local mass transfer that was necessary to account for convection mass losses 
during motor and blower start up and shut down, and while the local and overall measurements were conducted.

\section{DATA REDUCTION}

The local mass transfer coefficient is evaluated as

$$
h_{\mathrm{m}}=\dot{M}^{\prime \prime} /\left(\rho_{\mathrm{w}}-\rho_{\mathrm{b}}\right) .
$$

The mass flux, $\dot{M}^{\prime \prime}$, at each measurement point is evaluated from the density of solid naphthalene, the change of elevation during a test run, and the duration of the test run, after applying the correction that is necessary to account for the mass losses at the beginning and the end of the test run.

$$
\dot{M}^{\prime \prime}=\rho_{\mathrm{s}} \Delta z / \Delta t .
$$

In Eq. (1), the naphthalene vapor density at the wall, $\rho_{\mathrm{w}}$, is calculated using the ideal gas law along with the vapor pressure-temperature relation for naphthalene according to Eq. (1) in Ambrose et al. (1975). Since the naphthalene vapor density at the wall is constant, the boundary condition on the naphthalene surfaces in this investigation corresponds to the thermal boundary condition of uniform wall temperature.

The bulk density at the inlet of the test section is zero. The bulk density at the test section exit is obtained from the weights of the test section walls at the beginning and at the end of a test run, and the volumetric flow rate. The bulk density of naphthalene in the air stream, $\rho_{\mathrm{b}}$, at any streamwise location is then determined as a linear function of the streamwise coordinate using the bulk density values at the inlet and exit of the test section.

The Sherwood number is

$$
S h=h_{\mathrm{m}} D / \Lambda \text {. }
$$

The diffusion coefficient for naphthalene vapor in air, $\Lambda$, is calculated with Eq. (1) in Goldstein and Cho (1995). The corresponding Schmidt number is about 2.28. The Sherwood number is normalized by the corresponding Sherwood number for fully developed turbulent flow through a stationary smooth tube, $S h_{0}=0.023 R e^{0.8} S^{0.4}$.

Since rotation, sharp turns, and ribs affect the temperature and concentration fields, in a rotating multipass channel, in the same manner - through the velocity field, the following generalized heat/ mass transfer analogy may be employed,

$$
N u / N u_{0}=S h / S h_{0},
$$

where $N u_{0}=0.023 \operatorname{Re}^{0.8} \mathrm{Pr}^{0.4}$. Therefore, the normalized Sherwood number in this study may be considered as the comparison between the heat transfer coefficient for turbulent flow in a rotating two-pass square channel and that for corresponding fully developed turbulent flow in a stationary tube with a hydraulic diameter equal to that of the square channel.

The Reynolds number and the rotation number, both of which are based on the channel hydraulic diameter, are defined as $\dot{m} / \mu D$ and $\Omega D / U$, respectively.

The uncertainty of the local mass transfer coefficient depends on the uncertainties of the local wall and bulk naphthalene vapor densities, and the net local mass flux. The bulk naphthalene vapor density at the test channel exit is found to be up to $35 \%$ of the wall naphthalene vapor density. In calculating the uncertainty of the bulk density at a streamwise location, a maximum deviation of $5 \%$ of the wall density is estimated from the linearly interpolated value. Based on the method described in Kline and McClintock (1953), the maximum uncertainty of the Sherwood number is estimated to be $12.2 \%$, which occurs at the test channel exit. The uncertainty of the Reynolds number is found to be $4.8 \%$.

\section{PRESENTATION AND DISCUSSION OF RESULTS}

The detailed local heat (mass) transfer distributions have been determined on the leading and trailing walls of the two-pass square test channel, under fifty different test conditions - for various rib 
configurations, rotation numbers, and Reynolds numbers. The results of most of the test runs have been reported in Park (1996). The results to be presented here are for selected test runs in the case of $60^{\circ}$ ribs, with $R e=5500$ and $0.0<R o<0.24$. These results for $60^{\circ}$ ribs will be compared with corresponding results in the cases of a smooth channel and a channel with transverse or $90^{\circ}$ ribs.

In this section, the local heat (mass) transfer distributions will be presented as contours of $S h /$ $S h_{0}$ on the leading and trailing walls of the two-pass square test channel. The local Sherwood number ratio is determined on the two principal walls, using a grid of 36 points between consecutive angled ribs (along four lines, on the wall, that are parallel to the rib axes) and a grid of 96 points at the turn. However, the finite size of the tip of the measuring probe of the depth gage would not allow local measurements very close to the edges of the walls and the bases of the ribs. The local results are not extrapolated to the edges of the walls and the bases of the ribs.

The spanwise or regional average Sherwood number ratios, $\overline{S h} / S h_{0}$, on the leading and trailing walls will be presented along a streamwise coordinate, $X / D$. For the two straight passes of the test channel, $0.0 \leq X / D \leq 7.0$ and $9.5 \leq X / D \leq 16.5$, the values of $S h / S h_{0}$ at the nine distributed points along each of the grid lines (that are parallel to the rib axes) are averaged. The average value is considered to be at the streamwise location of the midpoint of the line.

In the turn, the values of $S h / S h_{0}$ are averaged over three regions. There are 36 distributed measurement points in an upstream square region with an area of $D \times D, 24$ points in a middle rectangular region with an area of $0.5 D \times D$, and 36 points in a downstream square region with an area of $D \times D$. These regions are considered to be located at $X / D=7.5,8.25$, and 9.0.

In the presentation of the streamwise distributions of the regional mass transfer, open and darkened symbols indicate the Sherwood number ratios on the leading wall and the trailing wall, respectively.

\section{Regional Average Mass Transfer in Rotating Smooth and Rib-roughened Channels}

In Figs. 4 and 5, the streamwise variations of $\overline{S h} / S h_{0}$ on the leading and trailing walls of a rotating channel with $60^{\circ}$ ribs are compared with those on the walls of rotating channels with $90^{\circ}$ ribs and with all smooth walls, for $R e=5500$, and $R o=0.09$ and 0.24. In the rib-roughened channels, there are transverse or angled ribs between $X / D=$ 1.0 and $X / D=6.0$ on the leading and trailing walls in the first pass only.

Attention is first focused on the radial outward flow in the first pass. Figures 4 and 5 clearly show that the mass transfer is very high where the flow reattaches on the walls between two transverse or angled ribs, and that the ribs enhance the overall mass transfer on the leading and trailing walls. With rotation, the values of $\overline{S h} / S h_{0}$ on the trailing walls in the first passes of all three channels are higher than corresponding values on the leading walls. In each channel, Coriolis force in the radial outward flow pushes the high momentum core fluid toward the trailing wall with accompanying pressure gradient across the channel cross section. The pressure field forces the low momentum fluid near the inner and outer walls toward the leading wall, forming double vortices. The higher mass transfer on the trailing wall and the lower mass transfer on the leading wall are primarily due to the shift of the high-velocity, low-concentration core flow toward the trailing wall.

Angled ribs cause higher overall mass transfer on both the leading and trailing walls than transverse ribs. The peaks of the $\overline{S h} / S h_{0}$ distributions between ribs are higher in the angled rib case than in the transverse rib case. It has been shown that, in stationary channels, the secondary flows that are induced by angled ribs increase the mass transfer on the walls between consecutive ribs (Kukreja et al., 1993). The results here show that the same is true in a rotating channel, on both the leading and trailing walls. The effect of the rib-induced secondary flow on flow reattachment downstream of the angled ribs on the leading and trailing walls and the 


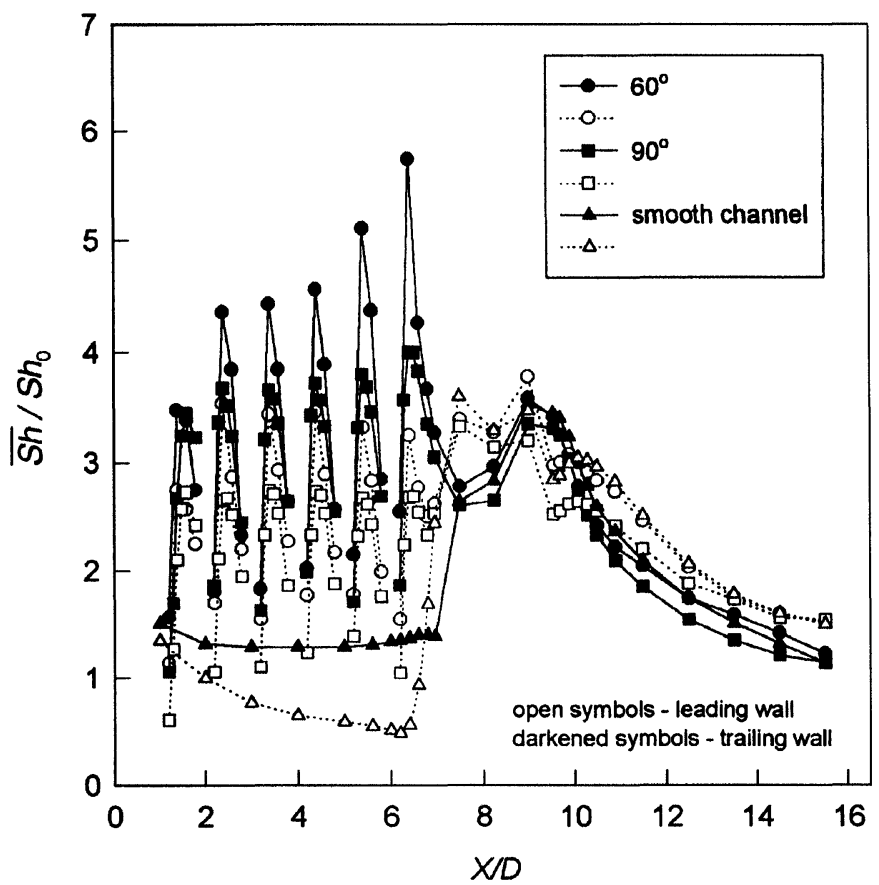

FIGURE 4 Streamwise variations of $\overline{S h} / S h_{0}$ in two-pass square channels with $60^{\circ}$ and $90^{\circ}$ ribs on the leading and trailing walls of the first passes, $R e=5500$ and $R o=0.09$.

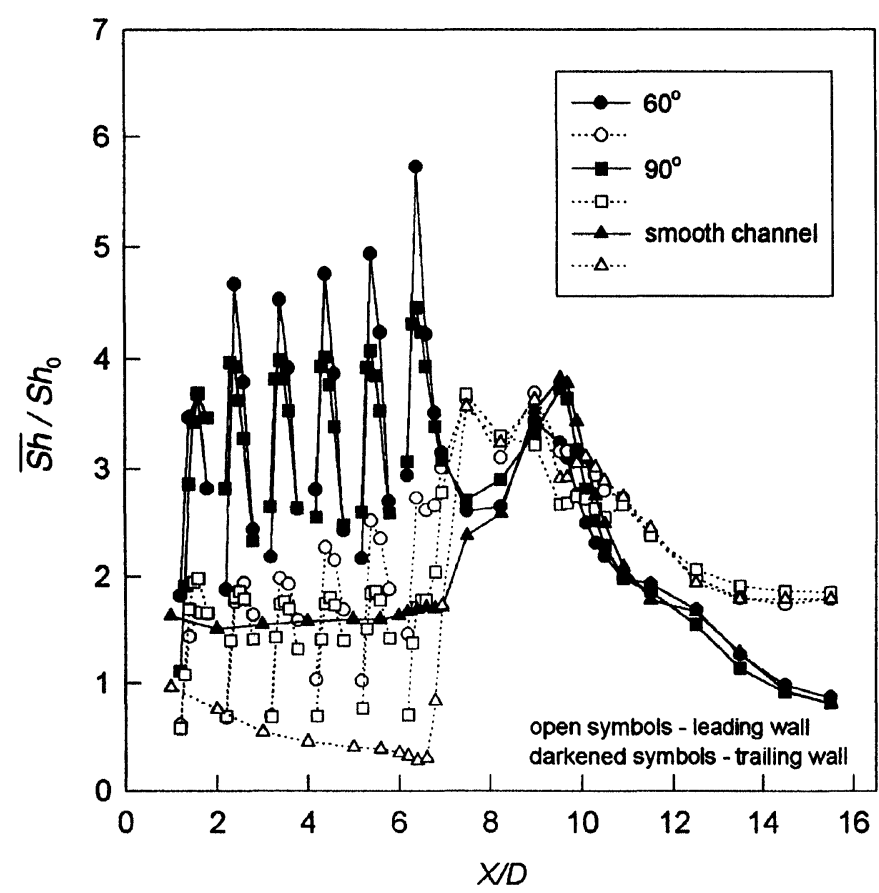

FIGURE 5 Streamwise variations of $\overline{S h} / S h_{0}$ in two-pass square channels with $60^{\circ}$ and $90^{\circ}$ ribs on the leading and trailing walls of the first passes, $R e=5500$ and $R o=0.24$ 
variation of $S h / S h_{0}$ on the walls between the angled ribs will be examined in a later section.

In the upstream region of the smooth turn (at $X / D=7.5$ ) in each of the three cases, the leading wall mass transfer increases abruptly and is higher than the trailing wall mass transfer. The high mass transfer on the leading wall is believed to be the result of the interaction between the rotationinduced vortices and the turn-induced vortices, which strengthens the turn-induced vortex nearer the leading wall, forcing much of the low-concentration core flow toward the leading wall.

In the downstream region of the turn (at $X /$ $D=9.0$ ), the mass transfer is high on both the leading and trailing walls. This may be caused by the emergence of a rotation-induced vortex pair, as the flow begins to turn radially inward. The vortex pair and the vigorous mixing of the separated flow downstream of the sharp inner corner may be responsible for the reduction of the imbalance of the strengths of the turn-induced vortex pair.

In the smooth second pass in each channel, the mass transfer on both walls decreases with distance from the turn, as the flow redevelops. With radial inward flow in the second pass, rotation increases the leading wall mass transfer and decreases the trailing wall mass transfer.

Figures 4 and 5 show that, the transverse and angled ribs in the first pass do not significantly affect the shapes of the mass transfer distributions on the smooth leading and trailing walls in the turn and in the second pass, suggesting that the flow field in a sharp turn and immediately downstream of a turn is, by and large, governed by the turn geometry rather than by the upstream disturbance.

\section{Overall Average Mass Transfer in Rotating and Stationary Channels}

The overall averages of the local Sherwood number values between the transverse and angled ribs at $X / D=4.0$ and 5.0 , and between the transverse and angled ribs at $X / D=5.0$ and 6.0 , on the leading and trailing walls in the first straight pass, for $R e=5500$ and $R o=0.09$ and 0.24 , are normalized with the corresponding overall averages for a stationary ribroughened channel. Similarly, the overall aver ses of the smooth wall Sherwood number values between $X / D=4.0$ and 5.0, and between $X / D=$ 5.0 and 6.0 , on the leading and trailing walls, with $R o=0.09$ and 0.24 , are normalized with the corresponding overall averages for a stationary smooth channel. These overall normalized Sherwood number values, $\overline{\overline{S h}} / \overline{\overline{S h}}_{\text {st }}$, are presented in Table I.

For the radial outward flow, rotation decreases the overall average mass transfer (relative to the stationary channel overall average mass transfer) on the leading wall of a smooth channel more than that on the leading wall of a rib-roughened channel. Rotation also increases the overall average mass transfer on the trailing wall of a smooth channel more than that on the trailing wall of a ribroughened channel. Rather unexpectedly, the $\overline{\overline{S h}} /$ $\overline{\overline{S h}}_{\text {st }}$ values for the $60^{\circ}$ rib case in Table I indicate that increasing the rotation number does not change the overall average mass transfer on the trailing wall of a channel with $60^{\circ}$ ribs. In a later section, it will be shown that rotation does not significantly change the local mass transfer distributions between consecutive $60^{\circ}$ ribs on the trailing wall.

TABLE I Overall normalized Sherwood number values, $\overline{\overline{S h}} / \overline{\overline{S h}}_{\text {st }}$, between $X / D=4.0$ and $X / D=6.0$, for channels with no ribs, $90^{\circ}$ ribs, and $60^{\circ}$ ribs

\begin{tabular}{|c|c|c|c|c|c|c|c|c|}
\hline \multirow[b]{3}{*}{$X / D$ range } & \multicolumn{4}{|c|}{$R o=0.09$} & \multicolumn{4}{|c|}{$R o=0.24$} \\
\hline & \multicolumn{2}{|c|}{ Leading wall } & \multicolumn{2}{|c|}{ Trailing wall } & \multicolumn{2}{|c|}{ Leading wall } & \multicolumn{2}{|c|}{ Trailing wall } \\
\hline & $4.0-5.0$ & $5.0-6.0$ & $4.0-5.0$ & $5.0-6.0$ & $4.0-5.0$ & $5.0-6.0$ & $4.0-5.0$ & $5.0-6.0$ \\
\hline Smooth channel & 0.58 & 0.55 & 1.22 & 1.31 & 0.40 & 0.38 & 1.50 & 1.61 \\
\hline $90^{\circ}$ ribs & 0.82 & 0.79 & 1.14 & 1.14 & 0.55 & 0.56 & 1.21 & 1.23 \\
\hline $60^{\circ}$ ribs & 0.76 & 0.69 & 0.97 & 1.01 & 0.53 & 0.55 & 1.02 & 0.98 \\
\hline
\end{tabular}




\section{Effect of Rotation}

To study the effect of rotation on the mass transfer on the leading and trailing walls of a rotating ribroughened channel, the $\overline{S h} / S h_{0}$ distributions in the first straight pass, for $R e=5500$ and $R o=0.0,0.09$, and 0.24 , are presented in Fig. 6. The $\overline{S h} / S h_{0}$ distributions in Fig. 6 show that increasing the rotation number decreases the $\overline{S h} / S h_{0}$ values on the leading wall, but does not significantly change the $\overline{S h} / S h_{0}$ values on the trailing wall. The. $\overline{S h} / S h_{0}$ distributions between consecutive ribs on both the leading and trailing walls are all similar, for $3.0<$ $X / D<6.0$. The trends of the spanwise average mass transfer distributions between consecutive ribs are consistent with the $\overline{\overline{S h}} / \overline{\overline{S h}}_{\text {st }}$ results presented in Table I.

Increasing the rotational speed causes larger velocity and concentration gradients near the trailing wall and smaller gradients near the leading wall, increasing the difference between the mass transfer on the two walls.
Figures 7 through 9 give the distributions of the local mass transfer for $R e=5500$, and $R o=0.0$, 0.09 and 0.24 , respectively. In the case of $R o=0.0$, the $S h / S h_{0}$ distributions on the leading and trailing walls are quite similar. With no rotation, the flow field and the concentration field should be symmetrical with respect to the midplane between the leading and trailing walls. Between angled ribs on both the leading and trailing walls of the first pass of the channel, the $S h / S h_{0}$ values are as high as over 5.0 near the inner wall and as low as below 2.0 near the outer wall. The large spanwise asymmetry of the $S h / S h_{0}$ distribution is the result of the secondary flow along the axes of the angled ribs, which sweeps the leading and trailing walls from the inner wall toward the outer wall. Since the angled ribs on the two opposite walls are parallel, the secondary flow induced by the angled ribs should be symmetrical with respect to the midplane between the two walls. This secondary flow may pull the main flow near the inner wall toward each ribbed wall, and may also force the separated flows over the ribs near the

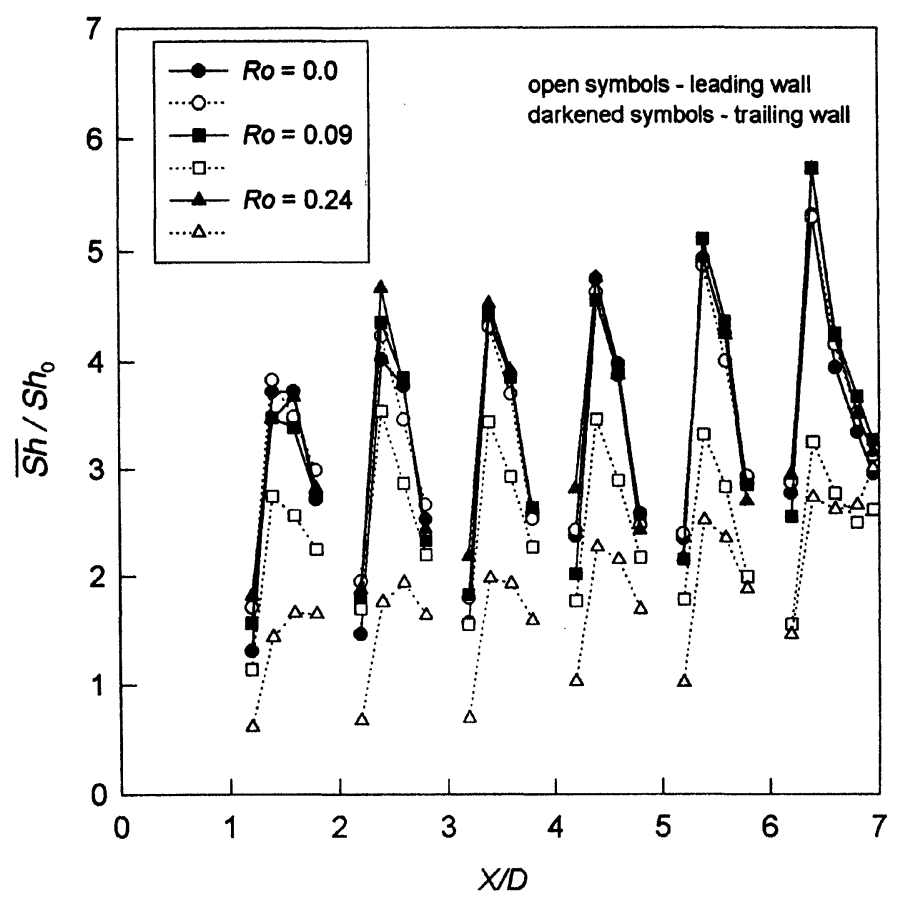

FIGURE 6 Effect of rotation on the streamwise variations of $\overline{S h} / S h_{0}$ in a square channel with $60^{\circ}$ ribs on the leading and trailing walls, $R e=5500$ and $R o=0.0,0.09$, and 0.24 . 


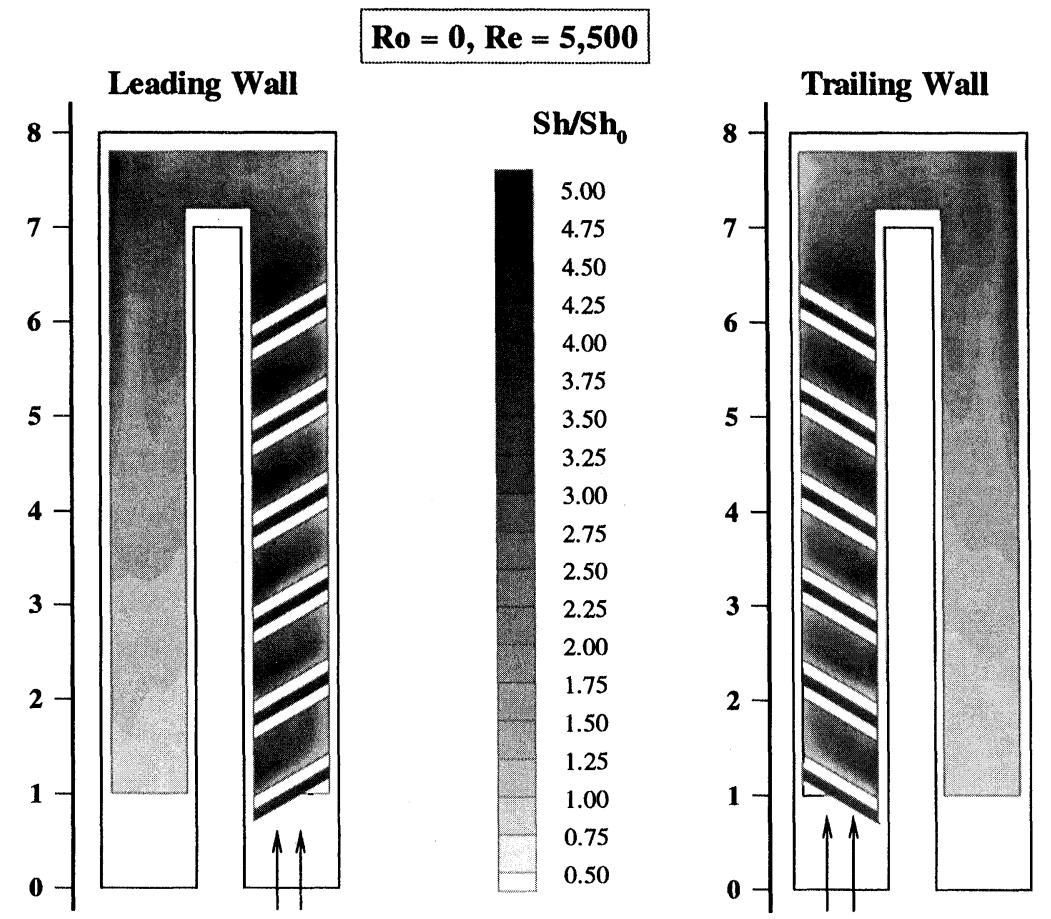

FIGURE $7 S h / S h_{0}$ distributions in a two-pass square channel with $60^{\circ}$ ribs on the leading and trailing walls of the first pass, $R e=5500$ and $R o=0.0$.

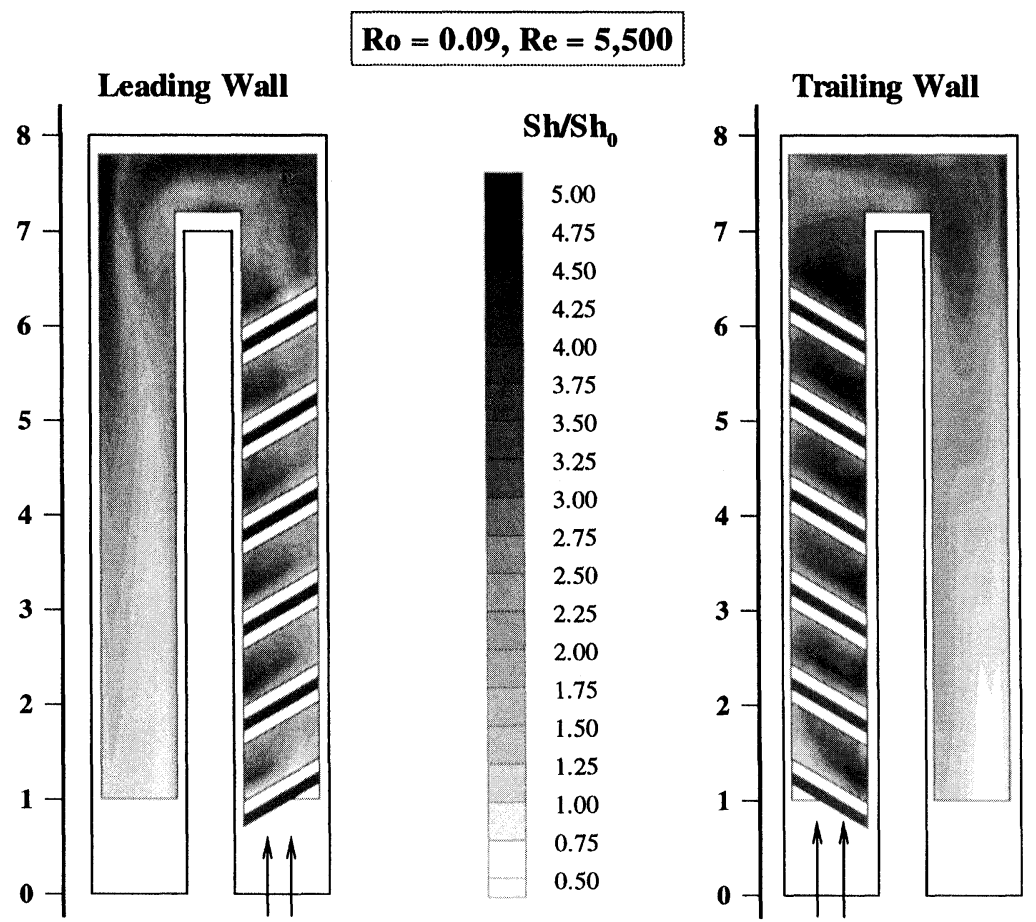

FIGURE $8 S h / S h_{0}$ distributions in a two-pass square channel with $60^{\circ}$ ribs on the leading and trailing walls of the first pass, $R e=5500$ and $R o=0.09$. 


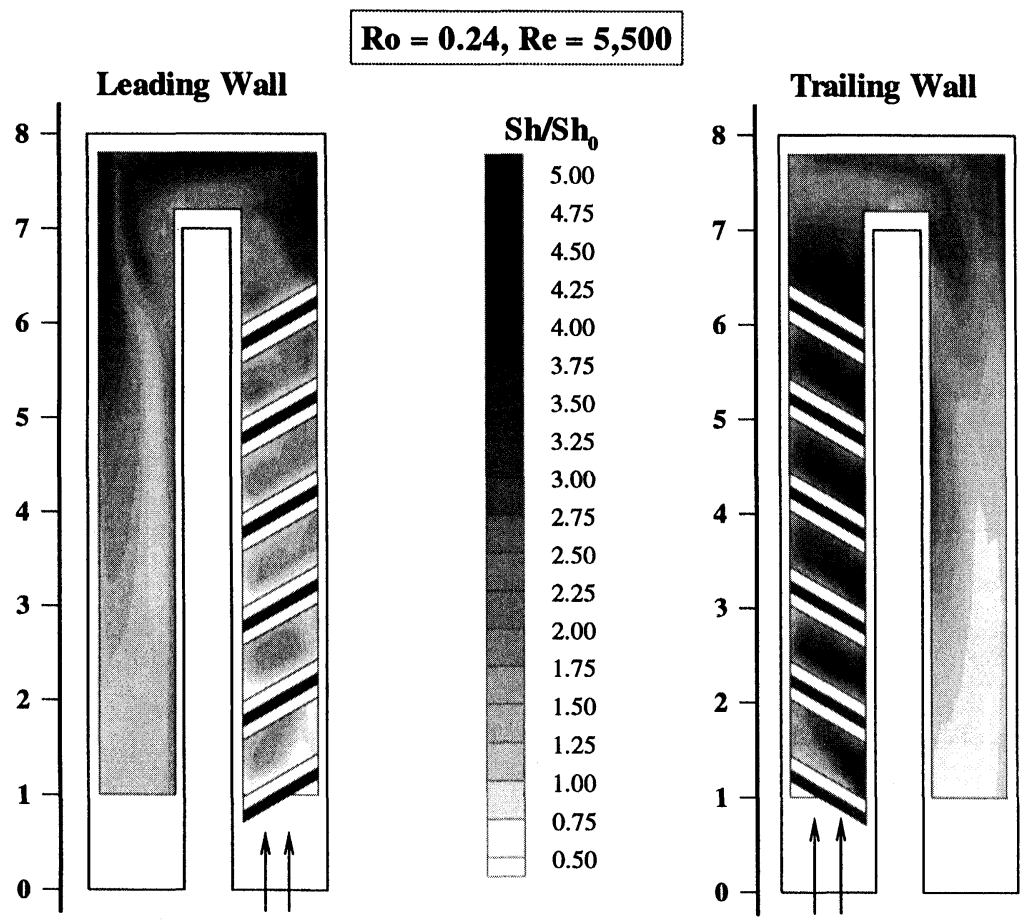

FIGURE $9 S h / S h_{0}$ distributions in a two-pass square channel with $60^{\circ}$ ribs on the leading and trailing walls of the first pass, $R e=5500$ and $R o=0.24$

inner wall toward the ribbed wall. Therefore, the $S h / S h_{0}$ values are very high near the inner wall.

As the rib-induced secondary flow moves along each wall between two ribs toward the outer wall, the secondary flow may weaken the reattachment of the separated flow over the ribs, resulting in the gradually decreasing $S h / S h_{0}$ values in the direction of the rib axes. Near the outer wall, the secondary flow may prevent the separated flow over the ribs from reattaching on the wall, as the secondary flow begins to turn away from the wall between two ribs. The large spanwise variations of $S h / S h_{0}$ between angled ribs are very different from the almost negligible spanwise variation of $S h / S h_{0}$ on the walls between transverse ribs (Park, 1996).

With rotation, $S h / S h_{0}$ decreases on the leading wall. The mass transfer distribution on the trailing wall, however, does not change appreciably (see also the $\overline{\overline{S h}} / \overline{S h}_{\text {st }}$ results in Table I). The spatially periodic flow separation and reattachment, and, in particular, the very strong near-wall secondary flow that are induced by the angled ribs in the high momentum region near the trailing wall, may lessen the propagation of the core flow toward the trailing wall, keeping the trailing wall mass transfer from increasing with rotation.

When $R o=0.09$, Fig. 8 shows that the shapes of the $S h / S h_{0}$ distributions between consecutive ribs on the leading wall are similar to those in the stationary case - decreasing mass transfer from the inner edge of the wall toward the outer edge. The $S h / S h_{0}$ values, however, are lower than corresponding values in a stationary channel. With rotation, the Coriolis force reduces the flow velocity near the leading wall. The weak reattachment of the low-momentum near-wall flows over the ribs also contributes to the low mass transfer between ribs on the leading wall.

The $S h / S h_{0}$ distributions between ribs on the leading wall for $R o=0.24$ are distinctly different from those for $R o=0.0$ and $R o=0.09$. Figure 9 shows that the mass transfer on the leading wall increases steadily along the streamwise direction in the first pass, with $S h / S h_{0}$ values much lower than those in the other two lower rotation number cases. 
The $S h / S h_{0}$ distributions between consecutive ribs do not have as large spanwise variations as those in the other two cases. Between $X / D=4.0$ and $X / D=6.0$, the $S h / S h_{0}$ distributions between consecutive ribs have twin peaks.

The leading wall mass transfer decreases with rotation, and, when the rotation number is relatively small, the local mass transfer on the leading wall decreases from the inner edge of the wall toward the outer edge, in the same manner as that on the trailing wall. The local mass transfer distribution on the leading wall may be dominated by the rib-induced secondary flow near the leading wall.

When the rotation number is large, Coriolis force distorts the velocity profile of the radial outward flow significantly. The rib-induced secondary flow near the trailing wall may be much stronger than that near the leading wall. Along the main flow direction, the strong secondary flow near the trailing wall may gradually strengthen the outer rotation-induced vortex, which has near-wall flow in the same direction as the secondary flow (toward the outer edge of the wall) near the trailing wall. This outer vortex may gradually dominate the inner vortex. The outer of the twin peaks in the local mass transfer distribution between two ribs downstream of the fourth rib on the leading wall in the $R o=0.24$ case (see Fig. 9) is believed to be the result of the reattaching flow near the outer edge of the leading wall being forced down by the strong outer vortex. The inner peak may be caused by the low-concentration core flow being circulated by the relatively weak inner vortex toward the inner portion of the leading wall.

The $S h / S h_{0}$ distributions in the turn and in the second pass with smooth walls that are presented in Figs. 8 and 9 are quite similar to those in channels with all smooth walls and with transverse ribs in the first pass only (Park, 1996). With increasing rotation number, the mass transfer along the outer edges of the leading wall in the turn increases significantly. The leading wall mass transfer near the inner corners of the turn is quite low. In the turn and immediately downstream of the turn, there is a smaller spanwise variation of the mass transfer distribution on the trailing wall than on the leading wall. Immediately downstream of the second inner corner of the turn, there is a relatively high mass transfer region that is also observed in rotating channels with all smooth walls and with transverse ribs. The mass transfer distributions on the smooth leading and trailing walls in the turn are caused by the interaction between the rotation-induced vortices and the turn-induced vortices, and flow separation and reattachment. The angled ribs on the leading and trailing walls in the first pass do not significantly affect the shape of the mass transfer distribution in the turn.

In the second pass, the mass transfer drops along the main flow direction, and the reversal of the Coriolis force for the radial inward flow causes the mass transfer to be higher on the leading wall than on the trailing wall.

\section{Effect of Ribs in Second Pass}

Figures 10 and 11 give the $\overline{S h} / S h_{0}$ and $S h / S h_{0}$ distributions, respectively, for $R e=5500$ and $R o=0.24$, in a channel with ribs on the leading and trailing walls of both the first and second passes. In Fig. 10, the $\overline{S h} / S h_{0}$ distributions for the corresponding transverse rib case are included for comparison.

For the radial inward flow in the second pass, the mass transfer is higher on the leading wall than on the trailing wall. However, the sharp turn reduces the difference between the mass transfer on the two walls, when compared with the difference in the first pass. As the flow redevelops, the spanwise average mass transfer is higher in the angled rib case than in the transverse rib case. Between two angled ribs on the leading and trailing walls in the second pass, the decreasing trend of the local mass transfer distributions toward the outer edges of the walls is similar to that in the first pass. Comparing Figs. 9 and 11, it is evident that the $S h / S h_{0}$ distributions in the turn are not affected by the angled ribs in the second pass. 


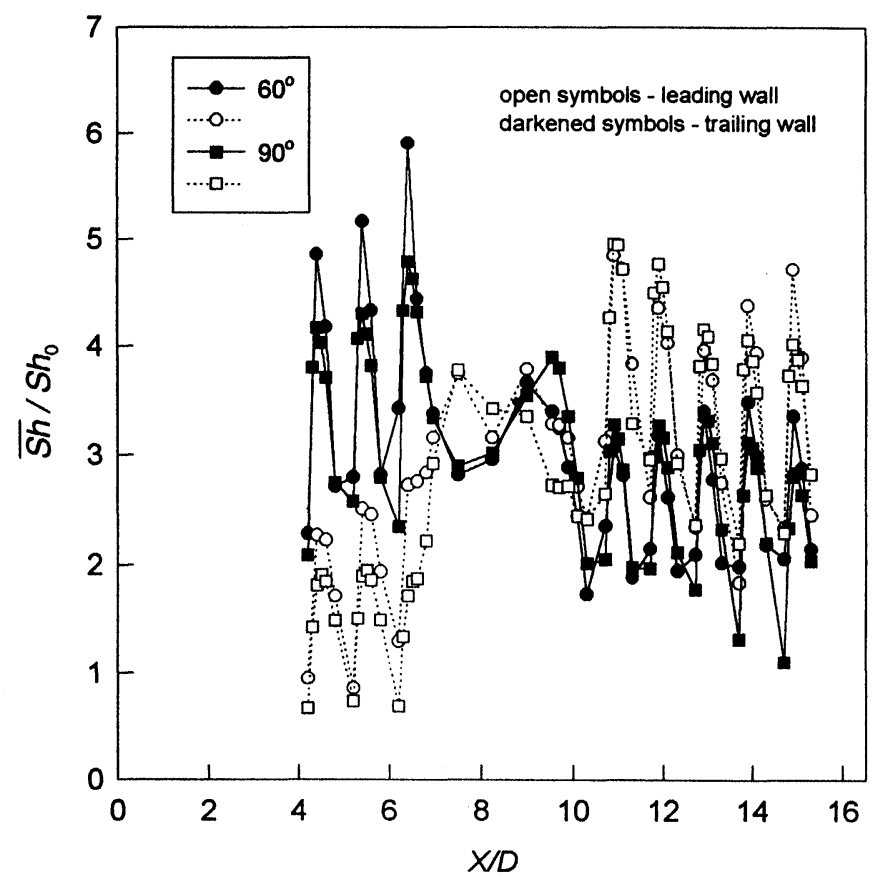

FIGURE 10 Streamwise variations of $\overline{S h} / S h_{0}$ in two-pass square channels with $60^{\circ}$ and $90^{\circ}$ ribs on the leading and trailing walls of both the first and second passes $R e=5500$ and $R o=0.24$.

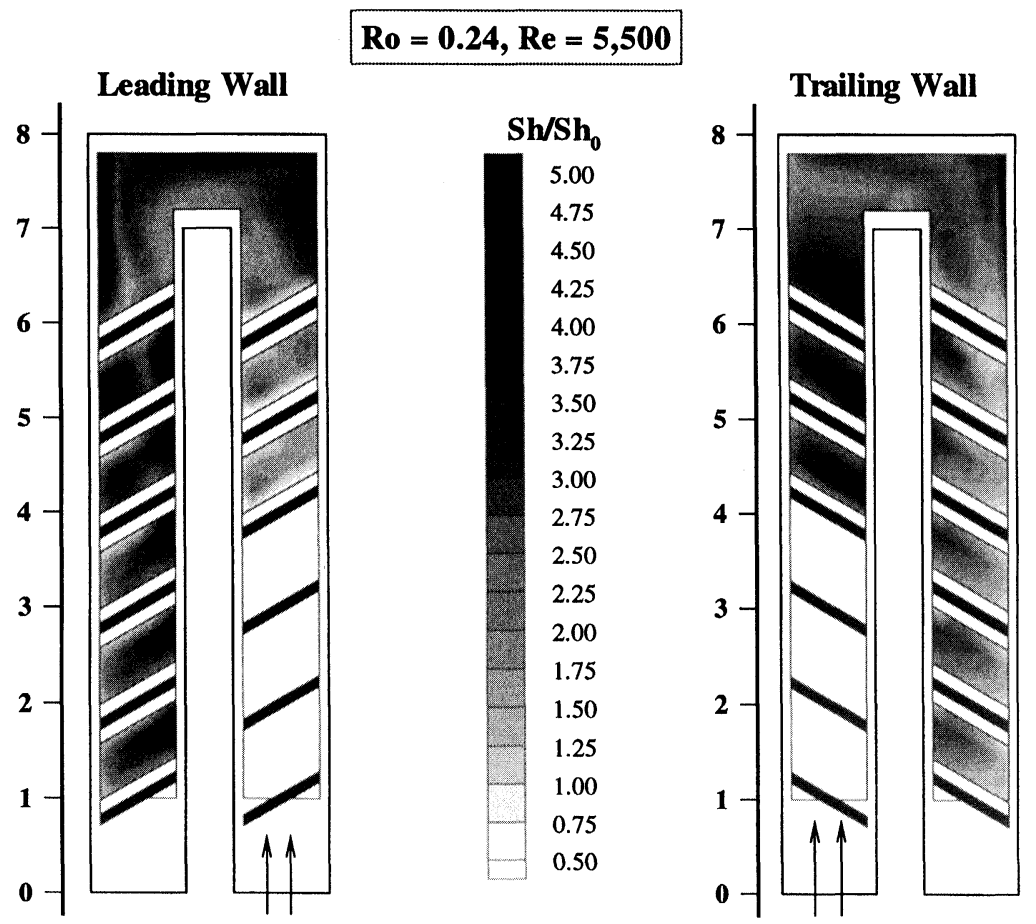

FIGURE 11 Local $S h / S h_{0}$ distributions in a two-pass square channel with $60^{\circ}$ ribs on the leading and trailing walls of both the first and second passes, $R e=5500$ and $R o=0.24$. 

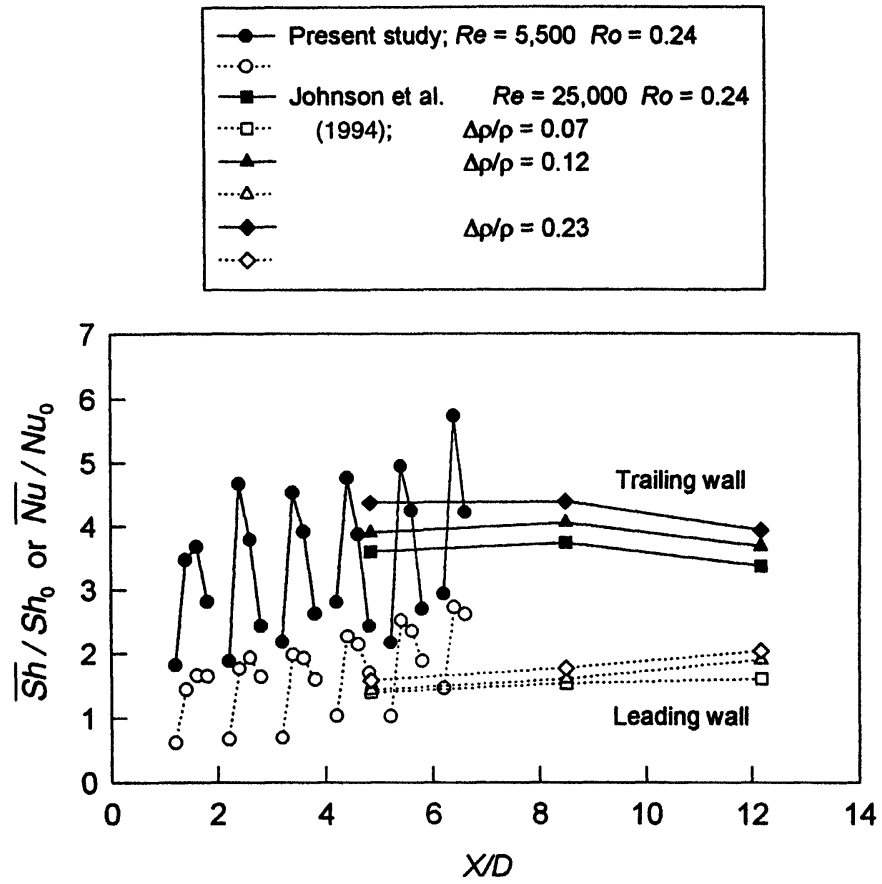

FIGURE 12 Comparison of the streamwise variations of $\overline{S h} / S h_{0}$ in this study with those of $\overline{N u} / N u_{0}$ from Johnson et al. (1994).

\section{Comparison with Published Results}

In Fig. 12 the $\overline{S h} / S h_{0}$ distributions on the leading and trailing walls of the first pass in the case of $R e=5500$ and $R o=0.24$ are compared with the corresponding $\overline{N u} / N u_{0}$ distributions by Johnson et al. (1994). The rotating channel in Johnson et al. (1994) had a very short unheated entrance channel, fourteen-hydraulic-diameter long straight passes, and a mean rotation radius of 49 times the hydraulic diameter. The $\overline{N u} / N u_{0}$ distributions in Fig. 12 are for $R e=25,000, R o=0.24$, and density ratio of $0.07,0.12$, and 0.23 . Despite differences in the test conditions of the two studies and negligible buoyancy variation effect in this study, the $\overline{S h} / S h_{0}$ distributions compare very well with the published $\overline{N u} / N u_{0}$ distributions.

\section{CONCLUDING REMARKS}

The effects of Coriolis force, sharp turn, and ribs on the local heat (mass) transfer distributions on the leading and trailing walls of a rotating, two-pass, square turbine blade cooling channel model with angled ribs have been investigated. Under the test conditions of the experiments reported here, the following conclusions may be drawn:

1. For radial outward flow in the first pass, rotational Coriolis force does not change the local or the overall heat (mass) transfer between consecutive angled ribs on the trailing wall.

2. Increasing the rotation number decreases the heat (mass) transfer on the leading wall. When the rotation number is high, there are twin peaks in the local heat (mass) transfer distribution between ribs on the leading wall.

3. For radial inward flow in the second pass, the heat (mass) transfer between consecutive ribs is higher on the leading wall than on the trailing wall. However, the sharp turn reduces the difference between the heat (mass) transfer on the leading and trailing wall. 
4. Comparing with the heat (mass) transfer distributions in a channel with smooth walls, the angled ribs on the leading and trailing walls of the two straight passes increase the variations of the heat (mass) transfer on the walls between two ribs, but do not significantly change the shapes of the local heat (mass) transfer distributions on the smooth walls in the turn.

5. Relative to the heat (mass) transfer in a corresponding stationary channel, the overall heat (mass) transfer in a rotating channel with angled ribs on the leading and trailing walls is not affected by the Coriolis force as much as that in rotating channels with smooth walls and with transverse ribs.

\section{NOMENCLATURE}

$C_{m} \quad$ cumulative mass of naphthalene in air stream $(\mathrm{kg} / \mathrm{s})$

D channel hydraulic diameter (m)

$h_{\mathrm{m}} \quad$ local mass transfer coefficient, Eq. (1) $(\mathrm{m} / \mathrm{s})$

$\dot{m} \quad$ mass flow rate of air $(\mathrm{kg} / \mathrm{s})$

$\dot{M}^{\prime \prime} \quad$ local mass flux on naphthalene surface, Eq. (2) $\left(\mathrm{kg} /\left(\mathrm{m}^{2} \mathrm{~s}\right)\right)$

$\mathrm{Nu} \quad$ local Nusselt number

$\overline{N u} \quad$ spanwise averaged Nusselt number

$N u_{0} \quad$ Nusselt number for fully developed flow, $=0.023 \operatorname{Re}^{0.8} \operatorname{Pr}^{0.4}$

$\operatorname{Pr} \quad$ Prandtl number

$\dot{Q} \quad$ volumetric flow rate of air $\left(\mathrm{m}^{3} / \mathrm{s}\right)$

$R \quad$ mean rotating radius (m)

$R e \quad$ Reynolds number based on channel hydraulic diameter, $=\dot{m} / \mu D$

Ro rotation number, $=\Omega D / U$

Sc Schmidt number of naphthalene in air, $\cong 2.28$

Sh local Sherwood number, Eq. (3)

$\overline{S h} \quad$ spanwise or regional average Sherwood number

$\overline{\overline{S h}} / \overline{\overline{S h}}_{\text {st }}$ overall average Sherwood number between two ribs, normalized with corresponding value for stationary channel
$S h_{0} \quad$ Sherwood number for fully developed flow, $=0.023 R e^{0.8} S c^{0.4}$

$U \quad$ average air velocity $(\mathrm{m} / \mathrm{s})$

$X \quad$ streamwise distance from mass transfer active channel inlet (m)

\section{Greek Symbols}

$\Delta t \quad$ duration of test run (s)

$\Delta z \quad$ elevation change at measurement point of naphthalene surface (m)

$\Delta \rho / \rho$ density ratio

$\Lambda$ diffusion coefficient of naphthalene vapor in air $\left(\mathrm{m}^{2} / \mathrm{s}\right)$

$\mu \quad$ dynamic viscosity of air $\left(\mathrm{Ns} / \mathrm{m}^{2}\right)$

$\Omega \quad$ angular velocity $(\mathrm{rad} / \mathrm{s})$

$\rho_{\mathrm{b}} \quad$ bulk naphthalene vapor density, $=C_{\mathrm{m}} / \dot{Q}$ $\left(\mathrm{kg} / \mathrm{m}^{3}\right)$

$\rho_{\mathrm{s}} \quad$ density of solid naphthalene $\left(\mathrm{kg} / \mathrm{m}^{3}\right)$

$\rho_{\mathrm{w}} \quad$ naphthalene vapor density at wall $\left(\mathrm{kg} / \mathrm{m}^{3}\right)$

\section{Acknowledgments}

This study was sponsored by NASA Lewis Research Center, Cleveland, Ohio (Contract Number: NAS3-27739) and the Center for Energy and Mineral Resources, College Station, Texas.

\section{References}

Ambrose, D., Lawrenson, I.J. and Sprake, C.H.S. (1975) The vapor pressure of naphthalene, Journal of Chemical Thermodynamics, 7, 1173-1176.

Goldstein, R.J. and Cho, H.H. (1995) A review of mass transfer measurements using naphthalene sublimation, Experimental Thermal Fluid Science, 10, 416-434.

Johnson, B.V., Wagner, J.H. and Steuber, G.D. (1993) Effects of Rotations on Coolant Passage Heat Transfer: Volume II Coolant Passages with Trips Normal and Skew to the Flow, NASA Contractor Report 4396, Vol. II.

Johnson, B.V., Wagner, J.H., Steuber, G.D. and Yeh, F.C. (1994) Heat transfer in rotating serpentine passages with trips skewed to the flow, Journal of Turbomachinery, 116, 113-123.

Kline, S.J. and McClintock, F.A. (1953) Describing uncertainties in single sample experiments, Mechanical Engineering, 75, 3-8.

Kukreja, R.T., Lau, S.C. and McMillin, R.D. (1993) Local heat/ mass transfer distribution in a square channel with full and $\mathrm{V}$-shaped ribs, International Journal of Heat and Mass Transfer, 36, 2013-2020.

Park, C.W. (1996) Local Heat/Mass Transfer Distributions in Rotating Two-Pass Square Channels, Ph.D. Dissertation, 
Department of Mechanical Engineering, Texas A\&M University, College Station TX

Park, C.W., Kandis, M. and Lau, S.C. (1998) Heat/Mass transfer distribution in a rotating two-pass square channel - Part I: Regional Heat Transfer, Smooth Channel, International Journal of Rotating Machinery (in press).

Parsons, J.A., Han, J.C. and Zhang, Y.M. (1994) Wall heating effect on local heat transfer in a rotating two-pass square channel with $90^{\circ}$ rib turbulators, International Journal of Heat and Mass Transfer, 37, 1411-1420.
Tse, D.G.N. and Steuber, G. (1996) Flow in Rotating Serpentine Coolant Passages with Skewed Trip Strips, NASA Contractor Report 198530.

Wagner, J.H., Johnson, B.V., Gruziani, R.A. and Yeh, F.C (1992) Heat transfer in rotating passages with trips normal to the flow, Journal of Turbomachinery, 114, 847-857.

Zhang, Y.M., Han, J.C., Parsons, J.A. and Lee, C.P. (1995) Surface heating effect on local heat transfer in a rotating twopass square channel with $60 \mathrm{Deg}$ angled rib turbulators, Journal of Turbomachinery, 117, 272-280. 


\section{ait \\ ENERGY MATERIALS}

M A N E Y publishing

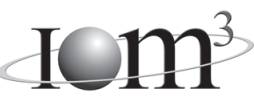

\section{Materials Science \& Engineering for Energy Systems}

Maney Publishing on behalf of the Institute of Materials, Minerals and Mining

The Institute of Materials, Minerals \& Mining

Economic and environmental factors are creating ever greater pressures for the efficient generation, transmission and use of energy. Materials developments are crucial to progress in all these areas: to innovation in design; to extending lifetime and maintenance intervals; and to successful operation in more demanding environments. Drawing together the broad community with interests in these areas, Energy Materials addresses materials needs in future energy generation, transmission, utilisation, conservation and storage. The journal covers thermal generation and gas turbines; renewable power (wind, wave, tidal, hydro, solar and geothermal); fuel cells (low and high temperature); materials issues relevant to biomass and biotechnology; nuclear power generation (fission and fusion); hydrogen generation and storage in the context of the 'hydrogen economy'; and the transmission and storage of the energy produced.

As well as publishing high-quality peer-reviewed research, Energy Materials promotes discussion of issues common to all sectors, through commissioned reviews and commentaries. The journal includes coverage of energy economics and policy, and broader social issues, since the political and legislative context influence research and investment decisions.

\section{CALL FOR PAPERS}

Contributions to the journal should be submitted online at http://ema.edmgr.com

To view the Notes for Contributors please visit: www.maney.co.uk/journals/notes/ema

Upon publication in 2006, this journal will be available via the Ingenta Connect journals service. To view free sample content online visit: www.ingentaconnect.com/content/maney

For further information please contact:

Maney Publishing UK

Tel: +44 (0)113 2497481 Fax: +44 (0)1132486983 Email: subscriptions@maney.co.uk

or

Maney Publishing North America

Tel (toll free): 8662975154 Fax: 6173546875 Email: maney@maneyusa.com

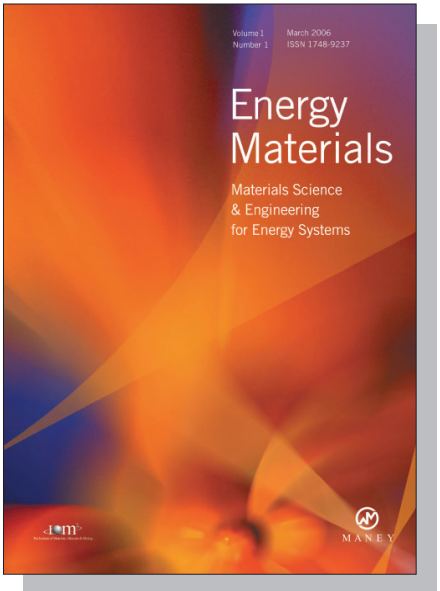

EDITORS

Dr Fujio Abe

NIMS, Japan

Dr John Hald, IPL-MPT, Technical University of Denmark, Denmark

Dr R Viswanathan, EPRI, USA

\section{SUBSCRIPTION INFORMATION}

Volume 1 (2006), 4 issues per year

Print ISSN: 1748-9237 Online ISSN: 1748-9245

Individual rate: $£ 76.00 / U S \$ 141.00$

Institutional rate: $£ 235.00 /$ US $\$ 435.00$

Online-only institutional rate: $£ 199.00 / U S \$ 367.00$

For special $\mathrm{IOM}^{3}$ member rates please email

subscriptions@maney.co.uk

\section{For further information or to subscribe online please visit www.maney.co.uk}



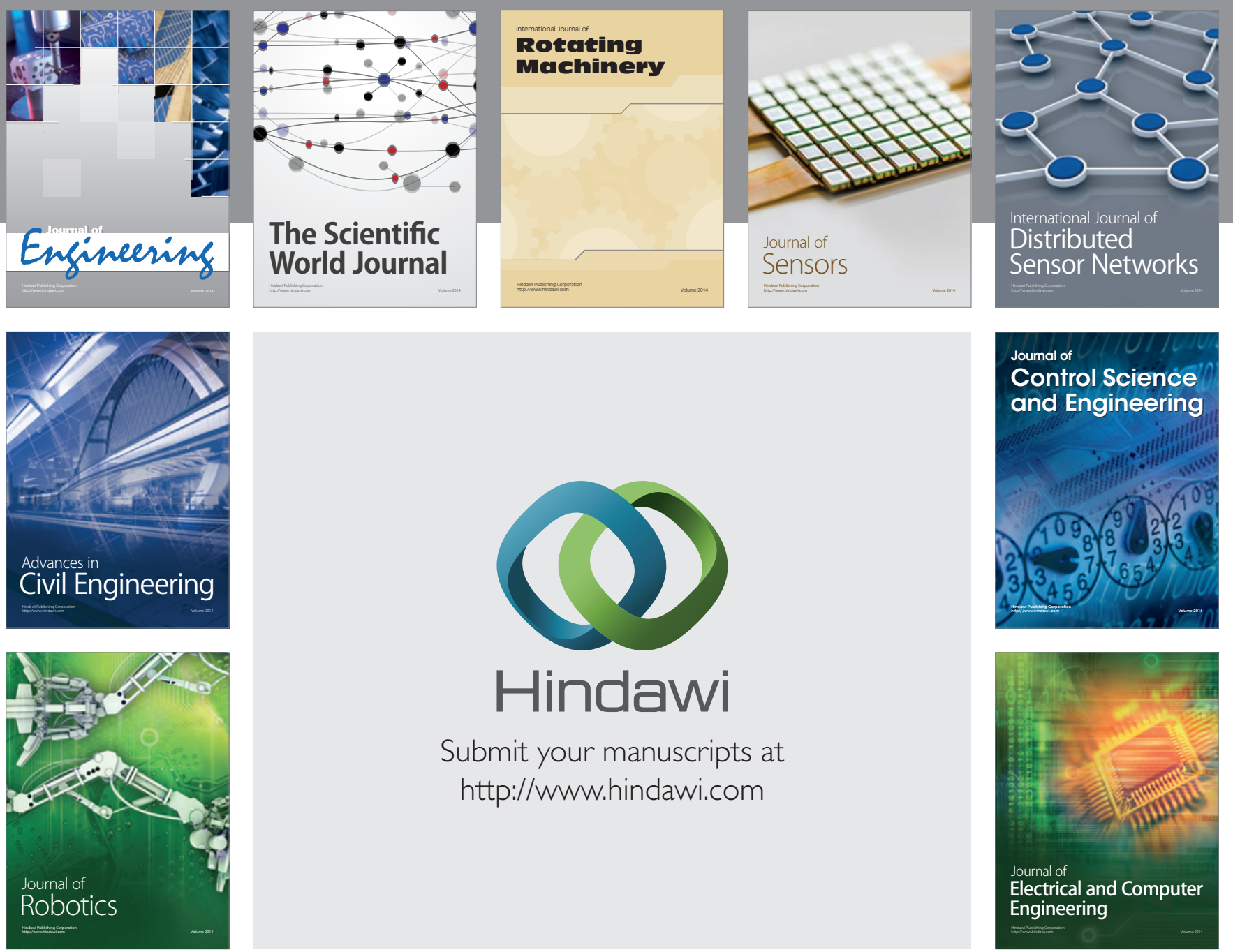

Submit your manuscripts at

http://www.hindawi.com
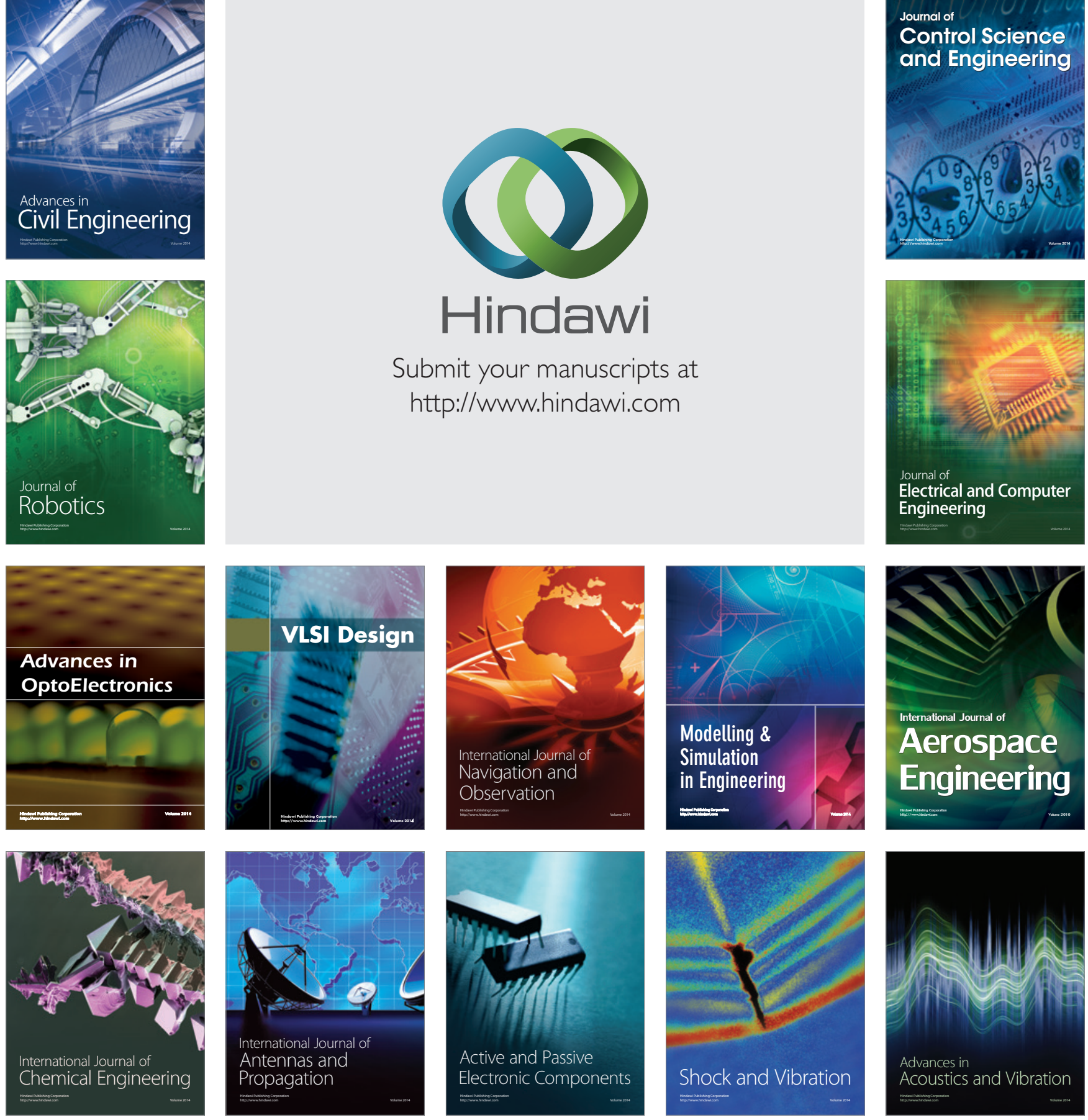